\title{
Survey of Registered Clinical Trials in Pregnant Women in China
}

yi zhao ${ }^{1}$, xiaofei luan ${ }^{1}$, hui yang ${ }^{2}$, guiping $\mathrm{du}^{1}$, qiongguang zhang ${ }^{3}$, zhengfu zhang ${ }^{3}$, and subiao wang ${ }^{4}$

1Zhejiang University School of Medicine Women's Hospital

${ }^{2}$ Device Evaluation Center Zhejiang Medical Products Administration

${ }^{3}$ Center for Food and Drug Inspection of NMPA

${ }^{4}$ Zhejiang Medical Products Administration

October 5, 2020

\begin{abstract}
Objective: To investigate the drug clinical trials in pregnant women in China to provide data on the quantity, properties, source of funding and geographical distribution for the registration and post-marketing studies. Methods: We collected data of 10865 clinical trials from the Drug Clinical Trial Registration and Information Disclosure Platform and 33649 clinical trials from Chinese Clinical Trial Registry (ChiCTR) performed on pregnant women between January 2013 and June 2020. Retained trials were analyzed based on several key variables (e.g., study endpoints, sources of funding, area of interest). Results: The percentage of trials regarding pregnancy registered to be carried out in China was $0.26 \%$, where the percentage of trials for new drug applications was $0.27 \%$. Most trials focused on anesthesia/analgesia, infections, and endocrine disorders. In the analyzed trials, most $(72.6 \%, 85 / 117)$ were designed using randomized parallel or cross-over grouping, and 59.8\% (70/117) of which put the health of the mother or fetal as the primary endpoint. There were no pre-planned pharmacokinetic studies in pregnant women. Only 5 new investigational drugs were developed for delivery and postpartum related treatments. The geographic distribution of the study sites was obviously uneven, with higher number of sites being distributed in the southeast of China (50 [41\%]). Conclusion: This survey showed that in China, there is a significant shortage in the development, evaluation and safety trial of pregnant drugs. Modifying or adding legislation and financial incentives may encourage the pharmaceutical companies to carry out clinical trials in pregnant women.
\end{abstract}

\section{Title page}

Title: Survey of Registered Clinical Trials in Pregnant Women in China

\section{Authors:}

1. Yi ZHAO (MD), Women's Hospital, School of Medicine, Zhejiang University

2. Xiaofei LUAN (MD),Women's Hospital, School of Medicine, Zhejiang University

3. Hui YANG (BA), Device Evaluation Center Zhejiang Medical Products Administration

4. Guiping Du (PhD), Women's Hospital, School of Medicine, Zhejiang University

5. Qiongguang ZHANG (PhD), Center for Food and Drug Inspection of NMPA

6. Zhengfu ZHANG $(\mathrm{PhD})$, Center for Food and Drug Inspection of NMPA

7. Subiao WANG (BA), Zhejiang Medical Products Administration

\section{Declaration of interests}

The authors report no conflict of interest.

\section{Acknowledgments}


Supported in part by the Zhejiang Provincial Natural Science Foundation (GF21F020055).

\section{Corresponding author:}

Deputy director Yi ZHAO, Department ofDrug Clinical Trials, Women's Hospital, School of Medicine, Zhejiang University, Hangzhou, Zhejiang, 310006, China. E-mail: zhaoyi1124@zju.edu.cn. Mobile phone number: 8613858004811.

Word count (abstract): 242

Word count (main text): 2986

\section{Cover Letter}

\section{Dear Editors:}

We would like to submit the enclosed manuscript entitled "Survey of Registered Clinical Trials in Pregnant Women in China ", which we wish to be considered for publication in "British Journal of Clinical Pharmacology".

In this work, we evaluated the first report of China about pregnant drug trials and provided robust data on the number, location, funding source and therapeutic area of drug trials in pregnancy. It was suggested that further research efforts should increase awareness among obstetricians and pregnant women and add legislative and fiscal incentives to inspire attention on pregnant drug clinical trials. I hope this paper is suitable for "British Journal of Clinical Pharmacology".

No conflict of interest exits in the submission of this manuscript, and manuscript is approved by all authors for publication. I would like to declare on behalf of my co-authors that the work described was original research that has not been published previously, and not under consideration for publication elsewhere, in whole or in part. Now the manuscript is submitted online, and I look forward to hearing from you.

Yours Sincerely,

Yi ZHAO

Department of Drug Clinical Trials,

Women's Hospital, School of Medicine, Zhejiang University, Hangzhou, Zhejiang, 310006, China.

Tel.: +86-13858004811

Fax: +86-571-89992206

Email: zhaoyi1124@zju.edu.cn

Abstract: Objective : To investigate the drug clinical trials in pregnant women in China to provide data on the quantity, properties, source of funding and geographical distribution for the registration and post-marketing studies. Methods : We collected data of 10865 clinical trials from the Drug Clinical Trial Registration and Information Disclosure Platform and 33649 clinical trials from Chinese Clinical Trial Registry (ChiCTR) performed on pregnant women between January 2013 and June 2020. Retained trials were analyzed based on several key variables (e.g., study endpoints, sources of funding, area of interest). Results : The percentage of trials regarding pregnancy registered to be carried out in China was $0.26 \%$, where the percentage of trials for new drug applications was $0.27 \%$. Most trials focused on anesthesia/analgesia, infections, and endocrine disorders. In the analyzed trials, most $(72.6 \%, 85 / 117)$ were designed using randomized parallel or cross-over grouping, and 59.8\% (70/117) of which put the health of the mother or fetal as the primary endpoint. There were no pre-planned pharmacokinetic studies in pregnant women. Only 5 new investigational drugs were developed for delivery and postpartum related treatments. The geographic distribution of the study sites was obviously uneven, with higher number of sites being distributed in the southeast of China $(50[41 \%])$. Conclusion : This survey showed that in China, there is a significant shortage in the 
development, evaluation and safety trial of pregnant drugs. Modifying or adding legislation and financial incentives may encourage the pharmaceutical companies to carry out clinical trials in pregnant women.

Keywords: Clinical trials, pregnant women, drug development, drug safety, drug innovation, sources of funding,geographical distribution, registration and post-marketing studies, legislation and financial incentives

\section{Backgroud}

The use of drugs during pregnancy is very common. Many pregnant women are being treated for existing or newly occurring medical conditions, including hypertension, diabetes, asthma, and psychiatry ${ }^{1}$; It is estimated that in the last three decades, $84 \sim 99 \%$ of women has taken medicines during pregnancy ${ }^{2-4}$. The pregnancy and delivery rate continue to rise after the implementation of the "two-child policy" in China. Statistical data from Shanghai showed that the proportion of severe pregnancies increased year-over-year from 2015 to 2018, and the incidence of pregnancy complications was up to $44.42 \%^{5}$. This may aggravate the problem of administration of drugs in pregnancy to some extent.

Over the past few decades, investments in the development of drugs for obstetric diseases have been very scarce. This severely limits the ability of obstetricians to make evidence-based prescriptions ${ }^{6}$. Therefore,bioethicist, pharmacologists, regulators, and researchers have advocated to inclusion of pregnant women in clinical trials to improve knowledge about the safety, dose, and long term effects of the drug in pregnant women $^{7-10}$. In 1993, the National Institutes of Health (NIH) recommended that pregnant women be included in the clinical trial ${ }^{11}$. The U.S. Food and Drug Administration (FDA) passed the Pregnancy and Lactation Labeling Rule (PLLR, Final Rule) in 2014 to replace traditional pregnancy drugs used by pregnant women. This law is expected to support further development and implementation of such drugs ${ }^{12}$. However, the insufficient number of pregnant women participating in clinical studies and being excluded from clinical trials are still common phenomena ${ }^{10,13}$. The study by Stockmann, et. al., reviewed the characteristics of obstetric studies registered at http://clinicaltrials. From 2007 to 2012, these studies accounted for less than $10 \%$ of the total number of registries ${ }^{14}$. A systematic evaluation in 2016 reported that in all valid registered drug clinical trials, only $0.32 \%$ were for pregnancy drugs, and even fewer (4.4\%) clinical trials included pre-planned pharmacokinetic studies ${ }^{15}$.

Although China has been working to develop regulations that comply with international standards, drug supply in mainland China is still affected by a backlog application or delay in the approval process until $2013^{16}$. In recent years, the National Medical Products Administration (NMPA) has implemented various measures to encourage the approval of special drugs (new chemical entities or drugs for the unmet medical need), and to accelerate the approval process of clinical trial of new drugs ${ }^{17}$. In order to further promote drug innovation and improve the efficiency of drug clinical trials, after 5 years revision, the Good Clinical Practice (GCP 2020) is enacted ${ }^{18}$, and the special protection requirements for pregnant women and fetuses were proposed in the Measures for Ethical Review of National Biomedicine Research $(2016)^{19}$. However, there were no detailed methods or plans in those document $\mathrm{s}^{20}$. So far, there is no publicly available systemic study regarding pregnancy drugs in mainland China. Therefore, we conducted a systematic survey of the clinical trial registration data to determine the quantity, geographical distribution, sources of funding and interested pharmacological areas of the clinical trials in pregnancy drugs between 2013 and 2020, so as to provide an up-to-date data as the reference for decision makers and other stakeholders to improve the current situation.

\section{Methods}

\section{Search strategy and selection criteria}

The clinical trial data registered in the Drug Clinical Trial Registration and Information Disclosure Platform (http://www.chictr.org.cn/) and ChiCTR (http://www.chinadrugtrials.org.cn) are collected ${ }^{21,22}$.Criteria for data inclusion: "valid" clinical trials between January 1, 2013 and June 30, 2020 involving medical intervention in pregnant women who intend to continue pregnancy. Trials completed in early 2013 and those having not finished enrollment in June 2020 were also included. Use the registry search function to search 
any of the following terms in the following names: "Obstetrics", "Pregnancy", "Pregnant", "Fetus", "Birth", "Perinatal", "Newborn", "Postpartum", "Prenatal", "Maternal", "Maternity", "Mother" or "Birth Outcome". This investigation included clinical trials of traditional Chinese medicines, supplements, and vitamins (figure 1).

\section{Data extraction and management}

Information publicly accessible on the website of NMPA includes: Sponsors and registered projects, such as funding organizations, types and locations; basic information of clinical trials, such as titles, study sites, date of first registration and insurance; study design, such as indications, name and category of drugs, study phase, design type, sample size, and primary and secondary endpoints. Except for the insurance, all other information is publicly accessible on the ChiTR website. The data of all of the above trial related information were extracted and compiled into an EXCEL worksheet for subsequent check and analysis.

\section{Data analysis}

All relevant trials are merged, checked and screened to exclude unqualified items. Microsoft EXCEL was used for descriptive statistics of the data, and then the trials were classified according to the following specific therapeutic areas (rank by quantity): anesthesia/analgesia; anti-infectiousagents (including treatment and prevention); endocrine diseases (mainly gestational diabetes mellitus (GDM) and thyroid diseases); postpartum hemorrhage (PPH) (only include the trials where subjects took the investigational drugs before giving birth); inducers; premature delivery/delivery; preeclampsia and pregnancy-induced hypertension (PIH); miscarriage and recurrent miscarriage; dietary supplement to improve pregnancy outcome; vomiting. The total number of trials in each database was compared with the number of trials relevant to pregnancy, and the comparison was expressed in percentage and original number. Analysis by region includes the location of the initiator and study sites. If the trial was conducted by different sites in the same region, it falls in the cumulative calculation for that region. Our analysis focused on three aspects of pregnancy trials: the overall finding of initial trials conducted between 2013 and 2020 (number, drug type, and study design); the therapeutic areas; and the geographical distribution of all clinical trial institutes and industry funds.

\section{Results \\ Overall findings}

As of June 30, 2020, the number of trials registered in the Drug Clinical Trial Registration and Information Disclosure Platform was 10,856, and the number of trials related to pregnancy drugs was 29 (0.27\%), of which only 6 trials recruited pregnant or parturient women. These trials included phase II and phase III, and the effectiveness and safety of these drugs for the mother were the endpoints. There were no pre-planned pharmacokinetic assays. The other 23 clinical trials where the investigational drugs' indications included pregnancy related diseases, but did not enroll any pregnant women. Interestingly, most of them $(18 / 23)$ were bioequivalence (BE) test and human pharmacokinetic test. There were 2 trials set the maternal or fetal outcomes as the primary endpoint. All trials on this platform used randomized parallel or cross-over grouping design. The number of trials registered in the "ChiCTR" was 33,649, and the number of trials enrolling pregnant women was $88(0.26 \%)$. All of them were post-marketing studies, including observational studies or interventional studies. Of the 88 studies, $63.6 \%$ (56/88) were randomized parallel controls, $17.0 \%(15 / 88)$ were cohort studies, 4 studies were cross-sectional designs, and $14.7 \%$ (13/88) did not indicate randomization. Of these trials, $77.3 \%(68 / 88)$ set both maternal and fetal outcomes as the primary endpoint. Annual numbers of pregnant drug clinical trials by study phase from those two registered platforms were shown in figure 2 .

\section{Clinical trial areas}

Merged data $(\mathrm{n}=117$ trials $)$ were analyzed to examine the levels of trial activity across therapeutic areas. As shown in Figure 3, the majority of the trials focused on three areas: anesthesia/analgesia (53 trials), anti-infective drugs (including 11 antibiotics; 8 antiviral agents), endocrine disorders (5 GDM, 5 thyroid dysregulation). Interestingly, the trials where new drugs were evaluated were focused on perinatal period: 
induction and enhancement of parturition (2), PPH (2), lactation (1), and constipation (1). Trials focusing on pre-eclampsia and PIH, vomiting, and thyroid autoimmune disease accounted for less than $4 \%$.

Detailed classification showed that in anesthesia/analgesia, the main study subjects were analgesia at delivery and prevention of caesarean section hypotension. In anti-infection, the most common subjects were antibiotics for gynecological infections and infection prevention for cesarean surgery. The number of trials in pregnant women with hepatitis B was the second highest. It is bizarre that there were few studies on TCM preparations. Only three trials were conducted for adjuvant treatment of postpartum constipation, postpartum lactation, and threatened abortion.It is noteworthy that there were 8 prospective pregnancy registries investigated the pregnancy outcomes following use of non-recommended medications or contraindicated medications such ribavirin, type-A toxin, doxycycline, and levofloxacin during and six months before pregnancy.

Unfortunately, the 5 newly tested drugs were merely the ones being structurally modified or with change in dosage form based on existing drugs. A total of $23.8 \%$ (21/88) of post-marketing trials evaluated the use of existing drugs in pregnant women that have not been approved by the FDA or NMPA for pregnancy use.

\section{Industry funding and study sites}

The sponsors and study regions between the post-marketing trials and the trials for new drugs are significantly different. Only 2 of these new drug trials were sponsored by foreign pharmaceutical companies, GlaxoSmithKline China and Shanghai Utimes Trading. The rest were mainly from domestic companies in Hubei (4 companies), Hebei (3), Hainan (3), Shanghai (3). 100 study sites were listed (Figure 4a), and 66\% $(66 / 100)$ were located in developed east cities and economical and cultural provinces such as Beijing (15), Shanghai (14), Jiangsu (13), Hunan (10), Zhejiang (7) and Shandong (7). Of the post-marketing clinical trials, only six were sponsored by pharmaceutical companies, $35.2 \%(31 / 88)$ were sponsored by society or foundation or the government, and the rest $57.9 \%$ were sponsored by the investigators themselves. The trials analyzed listed 82 institutions (Figure 4b), mainly located in east and south cities such as Zhejiang (13), Guangdong (12), Shanghai (10) and Jiangsu (10).

\section{Liability insurance of drug clinical trial}

Of the 29 new drug trials, $44.8 \%$ (13/29) of the sponsors purchased commercial insurance for subjects during the trial. Interestingly, these are trials evaluating BE and human pharmacokinetics. Such insurance is usually for compensate for injury or death related to the investigational drugs. In general, the cumulative liability limit is generally between RMB 300,000 and RMB 500,000 per person. Since ChiCTR platform does not require filling in relevant insurance information, it is unclear whether the insurance is purchased or not on the post-marketing studies.

\section{Discussion \\ Principal Findings}

In this clinical trial registration analysis, reliable data on the quantity, location, source of funding and therapeutic area of the trials on pregnancy related drugs were provided, thus to demonstrate that in China, pregnant women are the population that is also always excluded from clinical trials. To the best of our knowledge, this study is the first report regarding drug trials in pregnant women in China, although this finding is consistent with what reported by other foreign countries. All of these reports considered pregnant women as "drug orphan"23-25. Even when the number of pregnant women is sufficient, the number of clinical trials in pregnant women registered in China is very low compared to the regions and countries(e.g. North Africa/Middle East, Europe, America) where the trials on pregnancy related drugs are relatively active as indicated by J Scaffidi ${ }^{15}$. This may be related to study culture, sponsor's bias, and drug development trends.

In the past few decades, neither the investigators nor the patients were very enthusiastic to participate in clinical trials. Our study found that since the establishment of the NMPA registration platform in 2013, the total number of drug clinical trials registered on the platform and the CHiTR platform is 44,505 , which 
is only $1 / 5$ of the number of valid clinical trials on international registration platform within a year. Our study results also showed that the number of trials for new drugs and the assessments of efficacy and safety of the drugs for treatment of common pregnancy conditions are clearly insufficient. Similarly, there were no PK studies in pregnant women. This is possibly because that both the public and healthcare providers are worrying about the teratogenicity and other negative impact of the investigational drugs on the fetal development, rather than about the mother ${ }^{26}$. These data shows that the introduction of new drugs to pregnant women population will be complex. The mainstream prescribed drugs for pregnant women will still be off-label and the information, such as dosage, efficacy and safety for pregnant women, is insufficient.

\section{Research Implications}

\section{Risk and benefit}

Globally, there is a lack of representative studies on pregnancy drugs and pharmaceutical companies are not willing to develop new drugs or carry out clinical trials in pregnant women without explicit legislation or government incentives. Our findings suggested that the pharmaceutical industry being as sponsors accounts only for $10.3 \%(12 / 117)$, which is significantly lower than other pharmaceutical fields. This is consistent with the results of some previous analysis on sponsorship regarding pregnancy drug trials ${ }^{1,23,24}$. This also indicated that studies on pregnant women may require government's intervention, subsidy, incentive, and making advancement ${ }^{27}$. Our analysis also showed that almost all trials sponsored by pharmaceutical companies are for labor induction or post-partum adjuvant therapy. Possible litigation discourages them to use medicines indicated for general pregnancy conditions or for use in the first trimester of pregnancy. Similarly, it is not hard to see why this survey showed analgesia and anesthesia are the mostly focused therapeutic areas in pregnancy-related clinical trials. Apparently, the time of administration of these drugs and their effects on the fetus are short. Interestingly, the 5 new drugs under investigation are merely the ones being structurally modified, of with change in dosage form or in administration methods, which are the typical way of drug research and development the pharmaceutical companies adopt to avoid risks. Almost one-third of the trials only evaluated the marketed drugs for which a pregnancy prescription has not been approved. It was thought to be a way to reduce the investment and complicated process of new drug development. Similarly, purchasing drug clinical trial liability insurance is another way for the sponsors to avoid the risk of litigation and compensation.

\section{Policy support and trend}

In order to promote the research and development of innovative drugs in mainland China, the Chinese government has implemented the new priority review and approval procedure in 2015. Since 2016, the NMPA started evaluating the quality and efficacy of generic drugs in order to further reform and optimize drug innovation and improve the efficiency of drug clinical trials. In 2018, the 60-day Investigational New Drug Application System was officially adopted ${ }^{28-33}$. In 2020, the NMPA issued an announcement on three documents, including the review procedures for breakthrough therapeutic drugs, the review and approval procedures for the conditional approval of drug marketing applications, and the priority review and approval procedures for drug marketing authorization ${ }^{34}$. However, the results of our study suggested that there is no significant change in the number of trials regarding pregnancy drugs compared to the fast growing number of anti-cancer drug trials. From 2009 to 2018, the annual growth rate of anticancer drug trials in mainland China was $33 \%^{35}$. The long-term follow-up assessment and enrollment are challenging whereas the return and benefit are small, and there is a lack of established laws and regulations to promote the development of drugs for pregnant women. Prospective pregnancy registries to monitor drug-related adverse reactions and follow-up throughout pregnancy are a feasible approach. For example, the North American AntiEpileptic Drug (NAAED) Pregnancy Registry (http://www.aedpregnancyregistry.org/) and the National Pregnancy Registry for Antidepressants (NPRAD (https://womensmentalhealth.org/clinical and-researchprograms/) are the kind of website for registration and follow-up.In our study, we found six similar clinical studies in which the registered drugs were Levofloxacin, botulinus toxin, doxycycline, ribavirin, insulin and levothyroxine. It may be an important potential study direction in the future by establishing a collaborative research network for clinical trials to collect obstetric clinical practice information through electronic medical 
systems.

\section{Geographical distribution}

Our conclusion showed that the geographical distribution of study sites with pregnant women is significantly uneven, which is common in China. The study sites where many drug trials in pregnant women were conducted are tertiary hospitals in developed cities. This geographical difference was due to the uneven distribution of higher level of clinical trial institutions, rather than the uneven distribution of patients ${ }^{35}$. According to guidelines issued by NMPA on May 11, the clinical trial agency certification system will be replaced by a new records management system. Before carrying out a clinical trial at an eligible medical institution, simply register on the NMPA-authorized website. The purpose is to promote the formation of a highly positive medical innovation environment ${ }^{36}$. At the same time, the pharmaceutical companies, ethics committees and CROs will take more responsibility in the clinical trials.

\section{Strengths and Limitations}

The advantage of this study is to survey the clinical trial data of pregnant women registered on the clinical trial information platform in China by a systematic, unbiased approach. However, there are three major limitations. First, new drug clinical trials were required by NMPA to register on the drug clinical trial registration and information disclosure platform from September 6, 2013. Therefore, previously registered trials may not be included in the analysis. Second, ChiCTR was established in 2005 by West China Hospital of Sichuan University. In 2007, it was designated by the Ministry of Health to take part on behalf of China in the WHO International Clinical Trials Registry Platform. It is a non-profit academic institution and not a mandatory registry database ${ }^{7}$. Although some pharmaceutical companies or clinical institutions have conducted clinical trials in pregnant women, they have not yet registered on this information platform. Finally, there are 28,400 trials registered on ChiCTR that are still under review by ethics committee. Some of them were registered even back to 2007, but the registration status has not yet been updated. These are not included in our study because they do not meet the inclusion criteria for our study.

\section{Conclusions}

In summary, based on our findings, we think that the outlook on developing and evaluating new drugs for pregnant women may not be bright, unless there are appropriate policies and measures. However, the results of this study are not too frustrating either: In China, there are still studies conducted in pregnant women

at a certain frequency by sponsors and clinical institutions. More attention should be paid to the use of marketed drugs in pregnant women and give priority to the study on the PK of pregnant women.

\section{List of abbreviations}

\section{ChiCTR: Chinese Clinical Trial Registry}

FDA: the U.S. Food and Drug Administration

PLLR: the Pregnancy and Lactation Labeling Rule

NMPA: the National Medical Products Administration

GCP: the Good Clinical Practice

GDM: gestational diabetes mellitus

PPH: postpartum hemorrhage

PIH: pregnancy-induced hypertension

$\mathrm{BE}$ test: bioequivalence test

TCM: traditional Chinese medicine

PK study: pharmacokinetic studies 
NAAED: the North American Anti-Epileptic Drug

NPRAD: the National Pregnancy Registry for Antidepressants

\section{Contributors}

ZYand R-XFplanned and drafted the paper. YH, W-SBand D-GP contributed to data quality control, analysis, and interpretation.Z-QGandZ-ZFprovided administrative guidance and support with data interpretation. ZY led the overall planning and data interpretation. All authors reviewed and revised the manuscript.

\section{Declaration of interests}

We declare no competing interests.

\section{Acknowledgments}

Supported in part by the Zhejiang Provincial Natural Science Foundation (GF21F020055).

\section{References}

1. Sheffield JS, Siegel D, Mirochnick M, et al. Designing drug trials: considerations for pregnant women. Clin Infect Dis 2014; 59 Suppl 7: S437-44.

2. Mitchell AA, Gilboa SM, Werler MM, Kelley KE, Louik C, Herna 'ndez-D1 'az S. Medication use during pregnancy, with particular focus on prescription drugs: 1976-2008. Am J Obstet Gynecol2011;205(1):51.e1-8.

3. Haas DM, Gallauresi B, Shields K, et al. Pharmacotherapy and pregnancy: highlights from the third international conference for individualized pharmacotherapy in pregnancy. Clin Transl Sci2011;4(3):204-9.

4. Lupattelli A, Spigset O, Twigg MJ, et al. Medication use in pregnancy: a cross-sectional, multinational webbased study. BMJ Open 2014;4(2):e004365.

5. LI Guihua, HU Shuyi, SHEN Xinhe, ZHU Liping. Analysis on the characteristics of critical pregnant women in monitoring hospitals. Chinese Journal of Woman and Child Health Research2020;31(6):738-42.

6. Doering PL, Boothby LA, Cheok M. Review of pregnancy labeling of prescription drugs: is the current system adequate to inform of risks? Am J Obstet Gynecol 2002;187(2):333-9.

7. Lyerly $\mathrm{AD}$, Little MO, Faden $\mathrm{R}$. The second wave: toward responsible inclusion of pregnant women in research. Int J Fem Approaches Bioeth 2008;1(2):5-22.

8. Baylis F. Pregnant women deserve better. Nature2010;465(7299):689-90.

9. Johannes JM, Rieke G. Revised CIOMS International Ethical Guidelines for Health-Related Research Involving Humans. JAMA2017;317(2):135-136.

10. Shields KE, Lyerly AD. Exclusion of pregnant women from industrysponsored clinical trials. Obstet Gynecol2013;122(5):1077-81.

11. U.S. Department of Health \& Human Services. National Institutes of Health Revitalization Act of 1993. Science and Technology. Public Law. June 10, 1993; 103-43.

12. FDA. Pregnancy and lactation labeling final rule. December 3, 2014. Available from: https://www.fda.gov/vaccines-blood-biologics/biologics-rules/pregnancy-and-lactation-labeling-final-rule.

13. Cain J, Lowell J, Thorndyke L, Localio AR. Contraceptive requirements for clinical research. Obstet Gynecol 2000;95(6 Pt 1):861-6.

14. Stockmann C, Sherwin CMT, Koren G, et al. Characteristics and publication patterns of obstetric studies registered in ClinicalTrials. gov. J Clin Pharmacol 2014;54(4): 432-7.

15. Scaffidi J, Mol BW, Keelan JA. The pregnant women as a drug orphan: a global survey of registered clinical trials of pharmacological interventions in pregnancy. BJOG 2017;124(1):132-40. 
16. Zhao S, Lv C, Gong J, et al. Challenges in anticancer drug R\&D in China. Lancet Oncol 2019;20(1):18386.

17. Zhou Q, Chen XY, Yang ZM, Wu YL. The changing landscape of clinical trial and approval processes in China. Nat Rev Clin Oncol2017;14(9):577-83.

18. National Medical Products Administration\& National Heath Committee. Announcement on the issuance of quality management practices for drug clinical trials (no. 57 of 2020). April 26, 2020 (in Chinese). Available at: https://www.nmpa.gov.cn/xxgk/ggtg/qtggtg/20200426162401243.html.

19. The State Council. Order of the National Health and Family Planning Commission of the People's Republic of China on Measures for Ethical Review of National Biomedicine Research. October 12, 2016 (in Chinese). Available at:http://www.gov.cn/gongbao/content/2017/content_5227817.htm.

20. Jiang YF, Gen WQ, Zhu YH, Feng Y. The ethical protection for participants of vulnerable groups in clinical trials.MedicineEPPhilosophy 2017;38(6):25-7.

21. National Medical Products Administration. Announcement on the drug clinical trial information platform. September 6, 2013 (in Chinese). Available at: https://www.nmpa.gov.cn/yaopin/ypggtg/ypqtgg/20130906120001263.html.

22. World Health Organization. Primary registries, international clinical trials registry platform. July 25, 2007 (in Chinese). Available at: http://www.gov.cn/jrzg/2007-07/25/content_697236.htm.

23. Ayad M, Costantine MM. Epidemiology of medications use in pregnancy.Semin Perinatol 2015;39(7):50811.

24. McCormack SA, Best BM. Obstetric pharmacokinetic dosing studies are urgently needed. Front Pediatr $2014 ; 2: 9$.

25. Shields KE, Lyerly AD. Exclusion of pregnant women from industrysponsored clinical trials. Obstet Gynecol2013;122(5):1077-81.

26. Kass NE, Taylor HA, Anderson J. Treatment of human immunodeficiency virus during pregnancy: The shift from an exclusive focus on fetal protection to a more balanced approach. Am J Obstet Gynecol2000;182(4):856-9.

27. Mastroianni A, Kahn J. Swinging on the pendulum: Shifting views of justice in human subjects research. Hastings Cent Rep2001;31(3):21-8.

28. General Office of the State Council. State Council General Office's opinion on the evaluation of the consistency of quality and efficacy of generic drugs. March 5, 2016 (in Chinese). Available at: http://www.gov.cn/zhengce/content/2016-03/05/content_5049364.htm.

29. The State Council. State Council's opinions on reforming the examination and approval system for drug and medical devices. Aug 18, 2015 (in Chinese). Available at: http://www.gov.cn/zhengce/content/201508/18/content_10101.htm.

30. China Food and Drug Administration. Announcement of the General Administration on the work plan for the reform of the registration of chemical drugs (no. 51 of 2016). March 9, 2016 (in Chinese). Available at: https://www.nmpa.gov.cn/xxgk/ggtg/qtggtg/20160309151801706.html.

31. The General Office of the Central Committee of the Communist Party of China, the General Office of the State Council. The General Office of the Central Committee of the Communist Party of China and the General Office of the State Council issued the "Opinions on deepening the reform of the examination and approval system and encouraging the innovation of pharmaceutical medical devices". October 8, 2017 (in Chinese). Available at: http://www.gov.cn/zhengce/2017-10/08/ content_5230105.htm. 
32. National Medical Products Administration, National Health Commission. Announcement of the State Drug Administration and the State Health Commission on optimizing the examination and approval of drug registration and examination (no. 23 of 2018). May 23, 2018 (in Chinese). Available at: https://www.nmpa.gov.cn/zhuanti/ypqxgg/ggzhcfg/20180523110601517.html.

33. National Medical Products Administration. Announcement of the State Drug Administration on adjusting the approval process for drug clinical trial evaluation (no. 50 of 2018). July 27, 2018 (in Chinese). Available at: https://www.nmpa.gov.cn/xxgk/ggtg/ywlchshyjgrdgg/20180727172901286.html.

34. National Medical Products Administration. Announcement on the issuance of three documents including the "Working Procedures for Evaluation of Breakthrough Therapeutics (Trial)" (no. 82 of 2020). July 7, 2020 (in Chinese). Available at: https://www.nmpa.gov.cn/xxgk/ggtg/qtggtg/20200708151701834.html.

35. Ning Li, Hui-Yao Huang, Da-Wei Wu, et al. Changes in clinical trials of cancer drugs in mainland China over the decade 2009-18: a systematic review. Lancet Oncol 2019;20(11):e619-26.

36. National Heath Committee. Notice on printing and distributing the requirements for the work of the construction of the major clinical drug evaluation technology platform for major new drugs. Jan 21, 2019 (in Chinese). Available at: http://www.nhc.gov.cn/qjjys/s3593k/201901/f4b2ff724a564b35964b296b427b9002.shtml.

\section{Hosted file}

figure 1.pdf available at https://authorea.com/users/363938/articles/484556-survey-ofregistered-clinical-trials-in-pregnant-women-in-china

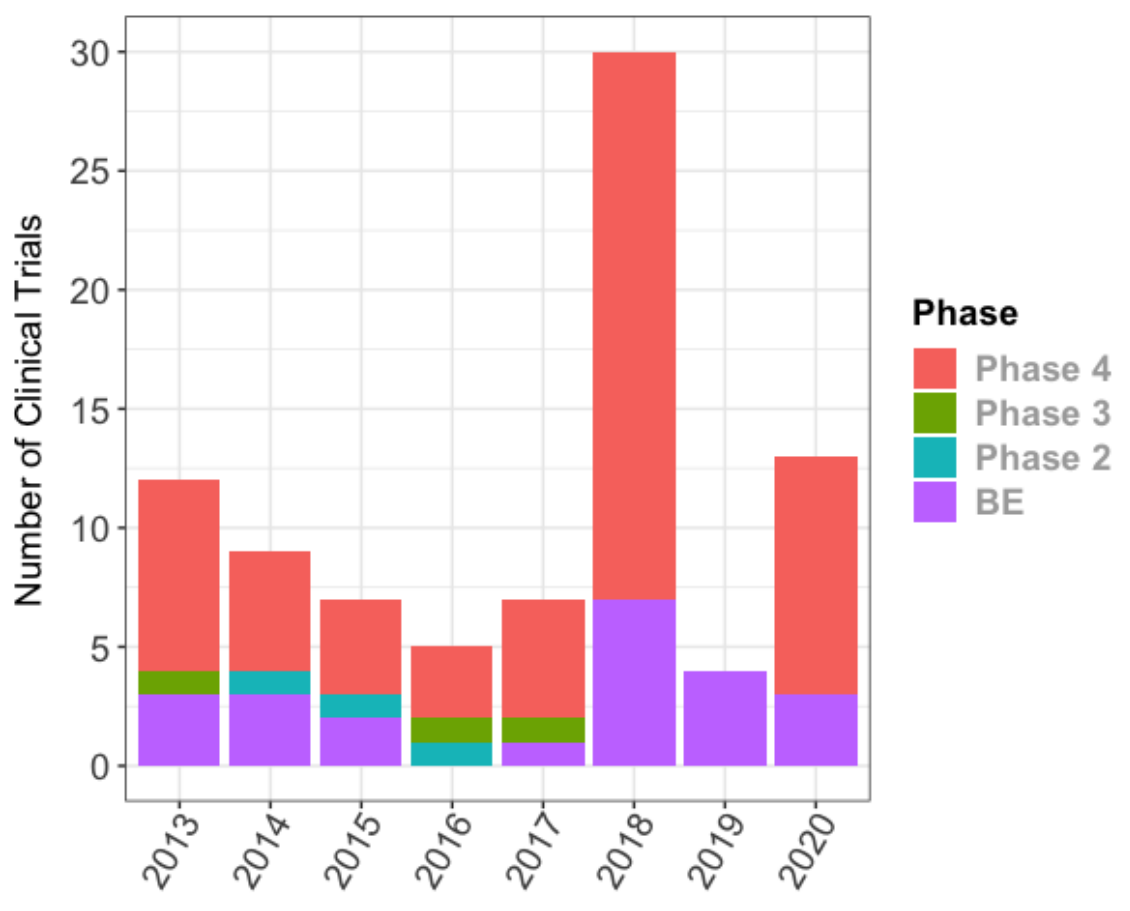


Anaesthesia \& Analgesia Anti-infectives

Endocrine Disorders (GDM and thyroid disorders) -

Prospective Pregnancy Registration Studies -

Pre-eclampsia \& $\mathrm{PIH}$ -

$\mathrm{PPH}$ -

Miscarriage \& Recurrent Pregnancy Loss

Varicose Veins -

Anemia -

Lactation -

Labour Induction \& Augmentation -

Hyperemesis -

Cardiovascular Diseases -

Cholestasis -

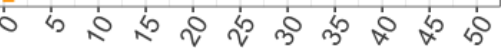

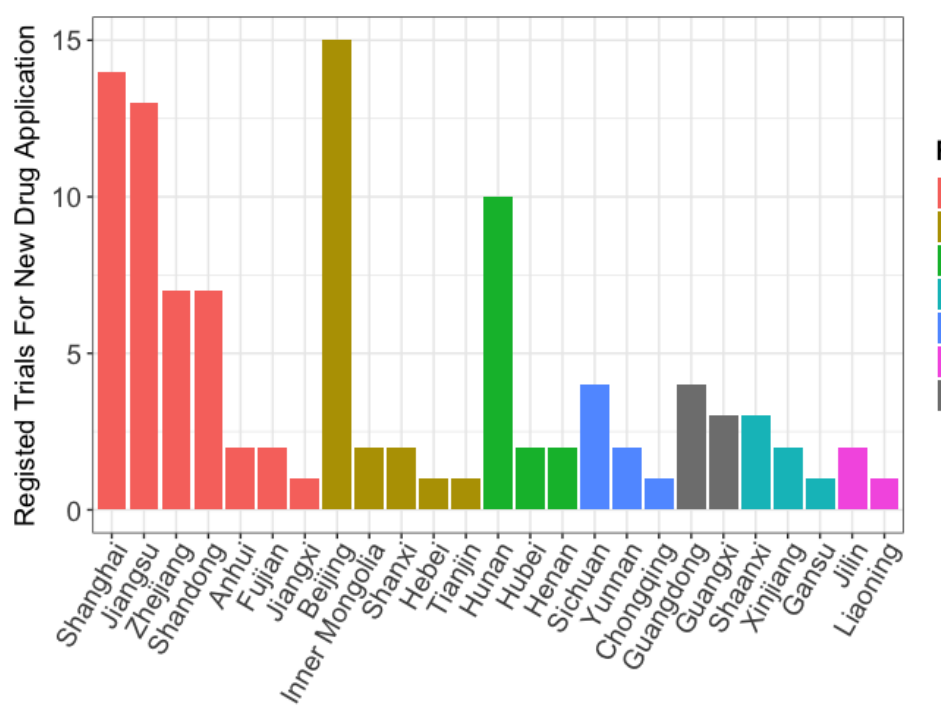

Region

East

North

Central

Northwest

Southwest

Northeast

NA 


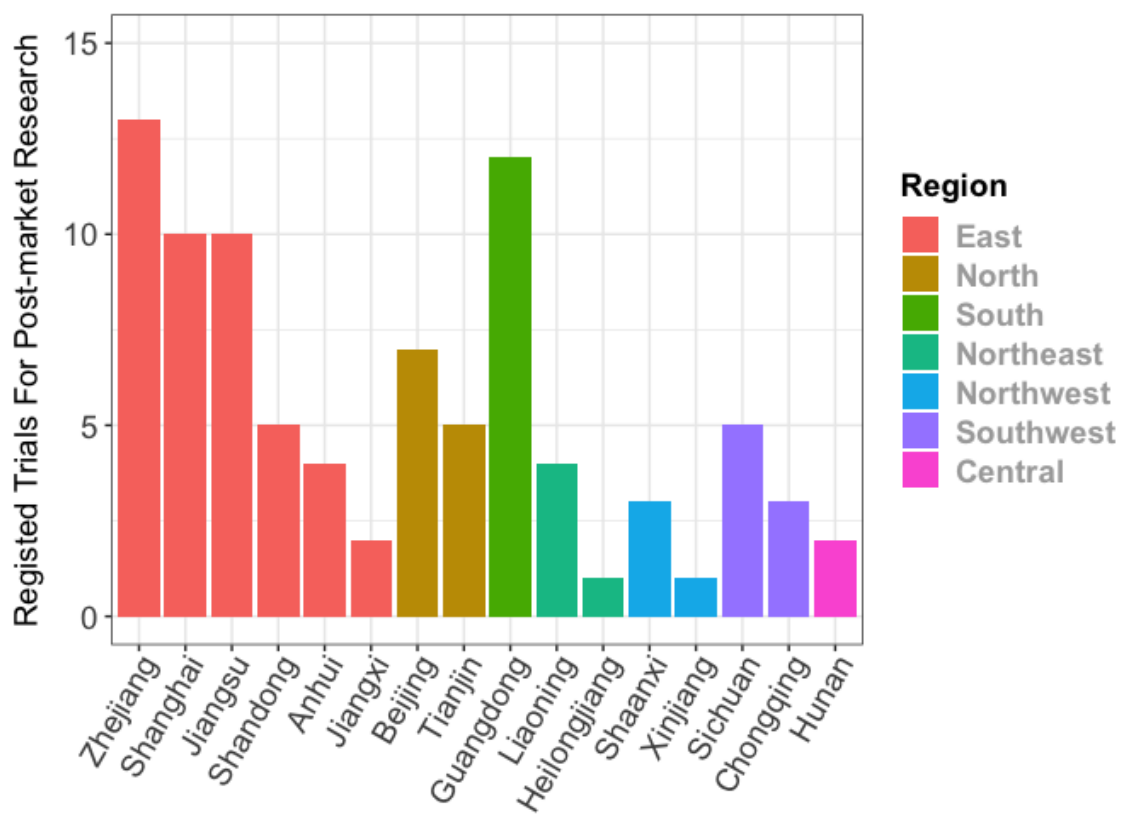

